\title{
Quantum-shell corrections to the finite-temperature Thomas-Fermi-Dirac statistical model of the atom
}

Burke Ritchie - Lawrence Livermore National Laboratory

\section{August 4, 2003}

U.S. Department of Energy

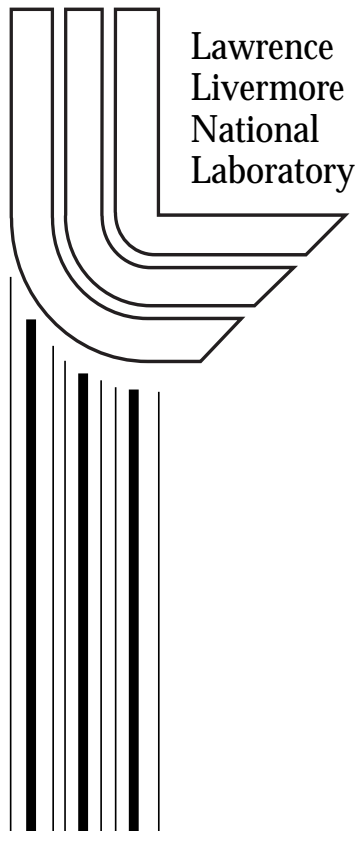


Quantum-shell corrections to the finite-temperature Thomas-Fermi-Dirac statistical model of the atom

\author{
Burke Ritchie \\ University of California \\ Lawrence Livermore National Laboratory \\ Livermore, CA 94550
}

Quantum-shell corrections are made directly to the finite-temperature Thomas-Fermi-Dirac statistical model of the atom by a partition of the electronic density into bound and free components. The bound component is calculated using analytic basis functions whose parameters are chosen to minimize the energy. Poisson's equation is solved for the modified density, thereby avoiding the need to solve Schroedinger's equation for a self-consistent field. The shock Hugoniot is calculated for aluminum: shell effects characteristic of quantum selfconsistent field models are fully captured by the present model. 
The finite-temperature Thomas-Fermi-Dirac (TFD) statistical model of the atom [1-2] has been extremely useful in calculating the equation of state (EOS) for hot, dense materials. Quantum selfconsistent-field (QSCF) models [3-5] followed as an improvement to TFD theory. The QSCF models however are plagued by uncertainties in boundary conditions and in convergence problems.

In this paper we make quantum shell corrections directly to the TFD model by a partition of the electronic density into bound and free components. The bound component is calculated using analytic basis functions whose parameters are chosen to minimize the energy. The local kinetic energy is calculated from the operation of the Laplacian on each basis function, divided by the basis function; contributions are retained only if the sum of the local kinetic and potential energies is negative. The free component is calculated from the standard TFD kinetic energy integral, which is modified by retention only of contributions in which the sum of the local kinetic and potential energies is positive. This procedure avoids double counting of bound and free contributions. The local potential energy is then calculated by solving Poisson's equation for the partitioned 
density, subject to the standard TFD boundary conditions. The exchange contribution is calculated using Slater's local exchange potential [3-4].

Next we present the mathematical development of the model. We solve Poisson's equation,

$$
\begin{gathered}
\nabla^{2} \mathrm{~V}=-4 \pi \mathrm{e}^{2} \rho_{\mathrm{m}} \\
\rho_{\mathrm{m}}=\Sigma \rho_{\mathrm{j}}^{(\mathrm{b})}+\int_{0}^{\infty} \mathrm{dx} \mathrm{x}^{\frac{1}{2}} \rho_{\mathrm{f}}(\mathrm{x}) \\
\rho_{\mathrm{j}}^{(\mathrm{b})}=\psi_{\mathrm{j}}(\overrightarrow{\mathrm{r}}) \mathrm{e}^{2}\left\{\mathrm{e}^{\left[\varepsilon_{\mathrm{j}}-(\mu-\mathrm{V}(\mathrm{r}))\right] / \mathrm{kT}}+1\right\}^{-1} \\
\rho_{\mathrm{f}}(\mathrm{x})=\frac{(2 \mathrm{mkT})^{\frac{3}{2}}}{2 \pi^{2} \hbar^{3}}\left[\mathrm{e}^{\mathrm{x}-(\mu-\mathrm{V}(\mathrm{r})) / \mathrm{kT}}+1\right] \\
\varepsilon_{\mathrm{j}}=-\frac{\hbar^{2}}{2 \mathrm{~m}} \frac{1}{\psi_{\mathrm{j}}} \nabla^{2} \psi_{\mathrm{j}}
\end{gathered}
$$

where in Eqs. (1b) and (1d) $x=\varepsilon / k T$ and in Eqs. (1c) and (1d) $\mu$ is the chemical potential [1-2]. Eq. (1a) is solved subject to the boundary condition $V=-Z / r$ near $r=0$ (atomic units used henceforth) and the constraint,

$$
\int \overrightarrow{d r} \rho_{m}(\vec{r})=Z
$$


In practical condensed-matter applications the atom is confined within an ion sphere [1-4] whose radius $r_{0}$ is determined from the reciprocal of the material number density or average volume per atom. Writing $V(r)=v(r) / r$ the boundary value $v(0)=-Z$ and the constraint given by Eq. (2), which guarantees electrical neutrality within the ion sphere, determine the boundary value of the derivative $v^{\prime}\left(r_{0}\right)=v\left(r_{0}\right) / r_{0}$ on the surface of the ion sphere; the chemical potential is given by $\mu=v\left(r_{0}\right) / r_{0}$, which ensures that the potential energy vanishes at $r_{0}$. It is convenient to integrate the radial equation backwards for trial values of $v\left(r_{0}\right)$ until Eq. (2) is satisfied. The basis-set members used in Eq. (1c) and (1e) (Table 1) are normalized within the ion sphere.

As pointed out in [1] the vanishing of the potential energy at $r_{0}$ means that the electronic pressure at $r_{0}$ is that for a free-electron gas, which is $2 / 3$ times the kinetic-energy density at $r_{0}$.

We further constrain the solution of Eq. (1a) by accepting contributions to the bound and free components of the electronic density [Eqs. (1c) and (1d) respectively] only if the sum of the local 
kinetic and potential energies satisfies the conditions $\left(\varepsilon_{j}+V\right) / k T<$ 0 in Eq. (1c) and $x+V / k T>0$ in Eq. (1d). The standard TFD model is recovered by dropping the bound component and accepting both negative and positive local total energy contribitions to Eq. (1d).

The set of basis functions indicated in Eqs. (1c) and (1e) are chosen to be Slater-type-orbitals (STO's), whose exponents (Table 1) are chosen to minimize the total energy at $\mathrm{kT}=0.026 \mathrm{EV}$ and normal density subject to the constraint given by Eq. (2). The total energy is given by,

$$
\begin{aligned}
& E=\int \overrightarrow{d r} T(\vec{r})-Z \int d \vec{r} \frac{\rho_{m}(\vec{r})}{r}+\frac{1}{2} \int d \vec{r} \int d \vec{r} \cdot \frac{\rho_{m}(\vec{r}) \rho_{m}\left(\overrightarrow{r^{\prime}}\right)}{\left|\vec{r}-\vec{r}^{\prime}\right|} \\
& \mathrm{T}=\mathrm{T}_{\mathrm{b}}+\mathrm{T}_{\mathrm{f}} \\
& \mathrm{T}_{\mathrm{b}}=\Sigma \varepsilon_{\mathrm{j}} \rho_{\mathrm{j}}^{(\mathrm{b})} \\
& \mathrm{T}_{\mathrm{f}}=\mathrm{kT} \int_{0}^{\infty} \mathrm{dx} \mathrm{x}^{\frac{3}{2}} \rho_{\mathrm{f}}(\mathrm{x})
\end{aligned}
$$

The solution of Eq. (1a) gives the partitioned density, which is used to calculate the energy. The parameters of the basis set are varied and the solution of Poisson's equation is repeated until the 
energy reaches a minimum. Numerical experiments suggest that a minimum principle exists for a cold material $(k T=0.026 \mathrm{eV})$ but not for a hot material $(\mathrm{kT}=10 \mathrm{eV})$; therefore the set of Slater-typeorbital (STO) parameters found to minimize the enrgy at $k T=0.026$ $\mathrm{eV}$ and normal density (Table 1 ) is used over the entire range of shock Hugoniot temperatures and densities apparently with satisfactory results (Figs. 1-2).

Since this solution self-consistently finds both the potential energy and the density, as in the standard TFD model, it avoids the need first to solve Schroedinger's equation for a self-consistent field in order to obtain the density. Quantum-shell effects however appear in the solution through the partition of the density into bound and free components. It is unclear if the upper bound on the energy of variational quantum mechanics is retained; however numerical experiments show that the total energy of the present model compares favorably with the total energy of the QSCF model, which typically is $25 \%$ higher than the total energy of standard TFD theory (Table 1). This result suggests that the partitioned density overcomes a defect of the TFD density, namely a singularity at the 
origin which is responsible for overestimating the binding energy.

A broad minimum near $-6.25 \mathrm{keV}$ is shown in Table 1. Energies versus $\zeta_{2 s}=\zeta_{2 p}$ eventually fall again for larger $\zeta_{2 s}=\zeta_{2 p}$; however Eq. (2) cannot then be satisfied. Moreover it is always possible to choose a set of parameters, for example one in which the subshell parameters are not equal, in which the energy is lower compared to the set shown in Table 1 and yet Eq. (2) is simultaneously satisfied; however these cases do not give sensible electronic densities, as in Fig. 1. It may be possible (although we have not investigated it) to choose a basis set, for example one in which the members are all mutually orthogonal as in the QSCF models, in which the present restriction that subshell parameters be equal is lifted. However the simplicity and accuracy of the present model compared to QSCF models is part of its appeal.

Our results are shown in Figs. 1-2. Fig. 1 shows the radial electronic density at $10 \mathrm{eV}$ and $1 \mathrm{~g} / \mathrm{cc}$ for $\mathrm{Al}$ for the TFD, the QSCF and the present quantum-shell corrected TFD (QSCTFD) models. We use the same computer code for all three models (Bulirsch- 
Storer automatic error control ODE solver [6]); in the QSCF model finite value and zero slope are taken for an orbital wave function and its derivative at $r_{0}$. At $10 \mathrm{eV}$ it is satisfactory to represent the continuum using several positive-eigenvalue orbitals from an original set of 12 orbitals of $s, p$, and d symmetries.

The QSCTFD result shows what appears to be a discontinuity near the L-shell maximum and a discontinuity and region of instability between 1 and 2 a. u. These artifacts are likely caused by the energy constraint on the partitioned density; nevertheless the model sensibly and robustly captures the shell structure of the atom (Figs. 1-2).

The oscillation of the QSCF result about the QSCTFD result above 2 a. $u$. in Fig. 1 reflects the quantum-continuum character of the former model. Nevertheless it seems satisfactory to represent the continuum contribution using the TFD prescription, as in the standard TFD model, in the present energy-constrained QSCTFD model, and, at least for the high-energy part of the contnuum, in our referenced QSCF models [3-5] .

Fig. 2 shows the shock Hugoniots for the various models 
compared with experimental data [7-12]. The INFERNO [4] result is taken from [5]. Since the publication of [5], an error has been discovered in the INFERNO code, whose correction gives a less compressible Hugoniot for high-Z materials; however this correction is not expected to be as important for low-Z materials [13].

In summary we have proposed and tested a modified form of the TFD statistical model of the atom, in which quantum-shell corrections are realized by partitioning the electronic density into bound and free components. Future work will include applications to high-Z materials.

Acknowledgements. The author is grateful for helpful comments and discussion on the EOS problem to Jim Albritton, John Castor, Dennis Maiden, Andy McMahan, Balazs Rozsnyai, Phillip Sterne and David Young. He is grateful to T. Scott Carman for support of the work. This work was performed under the auspices of the U.S. Department of Energy by the University of California Lawrence Livermore National Laboratory under Contract No. W-7405-ENG-48. 


\section{References}

1. R. P. Feynman, N. Metropolis, and E. Teller, Phys. Rev. 751561 (1949).

2. R. D. Cowan and J. Ashkin, Phys. Rev. 105144 (1957).

3. B. F. Rozsnyai, Phys. Rev. A 5137 (1.972).

4. D. A. Liberman, Phys. Rev. B 20, 4981 (1979).

5. B. F. Rozsnyai, J. R. Albritton, D. A. Young, V. N. Sonnad, and D. A. Liberman, Phys. Lett. A 291226 (2001).

6. J. H. Ferziger, Numerical Methods for Engineering Applications (Wiley, 1981), pp. 92-93.

7. V. A. Simonenko et al. Sov. Phys. JETP 61869 (1985).

8. A. P. Volkov et al. JETP Lett. 31588 (1980).

9. E. N. Avrorin et al. JETP Lett. 43309 (1986).

10. A. S. Vladimirov et al. JETP Lett. 3982 (1984). 
11. L. V. Al'tshuler et al. Sov. Phys. JETP 45167 (1977).

12. A. C. Mitchell et al. J. Appl. Phys. 692981 (1991).

13. J. R. Albritton (private communication). 
Table 1. Aluminum: total energies and variational parameters for the Slater-type-orbitals $\psi_{\text {nlm }}=N_{n l m}{ }^{n+1-1} e^{-\zeta_{n f}} Y_{l m}$ at $k T=0.026 \mathrm{eV}$ and a normal density of $2.7 \mathrm{~g} / \mathrm{cc}$. Filled subshells are automatically spherically symmetric and unfilled subshells are spherically averaged. At this level of simplicity the energy [Eq. (3a)] has a minimum and Eq. (2) is simultaneously satisfied if $\zeta_{\mathrm{nl}}=\zeta_{\mathrm{nl}}$.

$$
\begin{aligned}
& \zeta_{1 \mathrm{~s}}=13.0 \\
& \zeta_{2 \mathrm{~s}}=\zeta_{2 \mathrm{p}}=4.5 \\
& \zeta_{3 \mathrm{~s}}=\zeta_{3 \mathrm{p}}=2.0
\end{aligned}
$$

$E_{\text {QSCTFD }}=-6.25 \mathrm{keV}$ (present model)

$E_{Q S C F}=-6.55 \mathrm{keV}$ (Ref. 3)

$\mathrm{E}_{\text {TFD }}=-8.49 \mathrm{keV}$ (Ref. 3)

Example of the minimum energy versus $\zeta_{21}$ for fixed values shown above for the other parameters:

$\begin{array}{lc}\text { EQSCTFD } & \zeta_{\mathbf{2}}=\zeta_{\mathbf{2 p}} \\ -6.09 \mathrm{keV} & 3.5 \\ -6.25 \mathrm{keV} & 4.5 \\ -6.23 \mathrm{keV} & 5.5 \\ -6.03 \mathrm{keV} & 6.5\end{array}$




\section{Figure Captions}

Fig. 1 Radial electronic density for aluminum at $10 \mathrm{eV}$ and $1 \mathrm{~g} / \mathrm{cc}$.

The pressures calculated for the various models are $\mathrm{P}_{\mathrm{TFD}}=0.853 \mathrm{Mb}$,

$P_{Q S C F}=0.63 \mathrm{Mb}$ (converged to 2 places after 20 iterations), and PQSCTFD $=0.666 \mathrm{Mb}$.

Fig. 2. Shock Hugoniots for aluminum for the various models and from experiment. QEOS is based on the Thomas-Fermi model with "cold curve" empirical adjusments (Ref. 5). The QSCTFD result does not include the ion contribution, which is small and is included in the other models using the ideal gas law. 


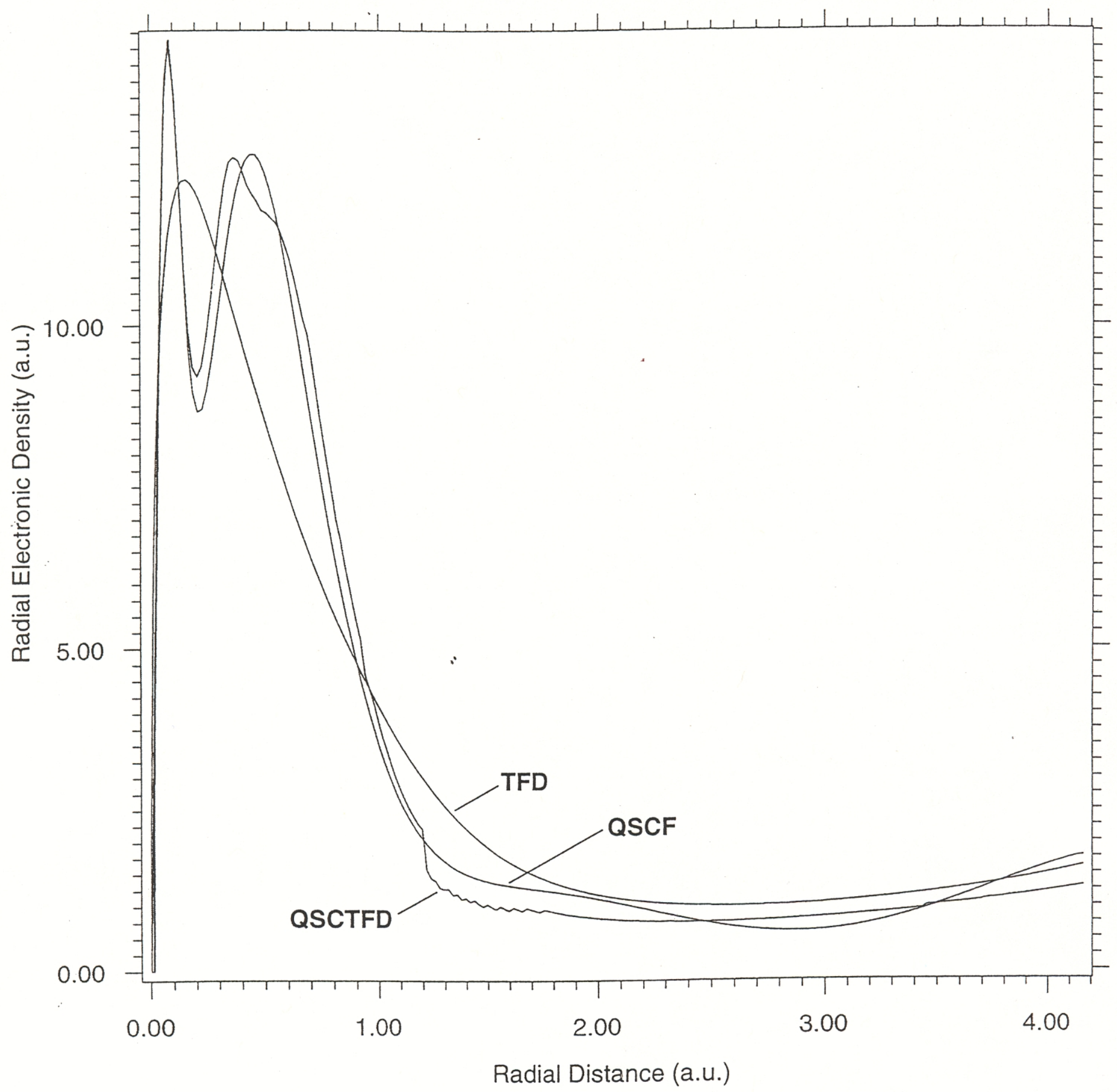




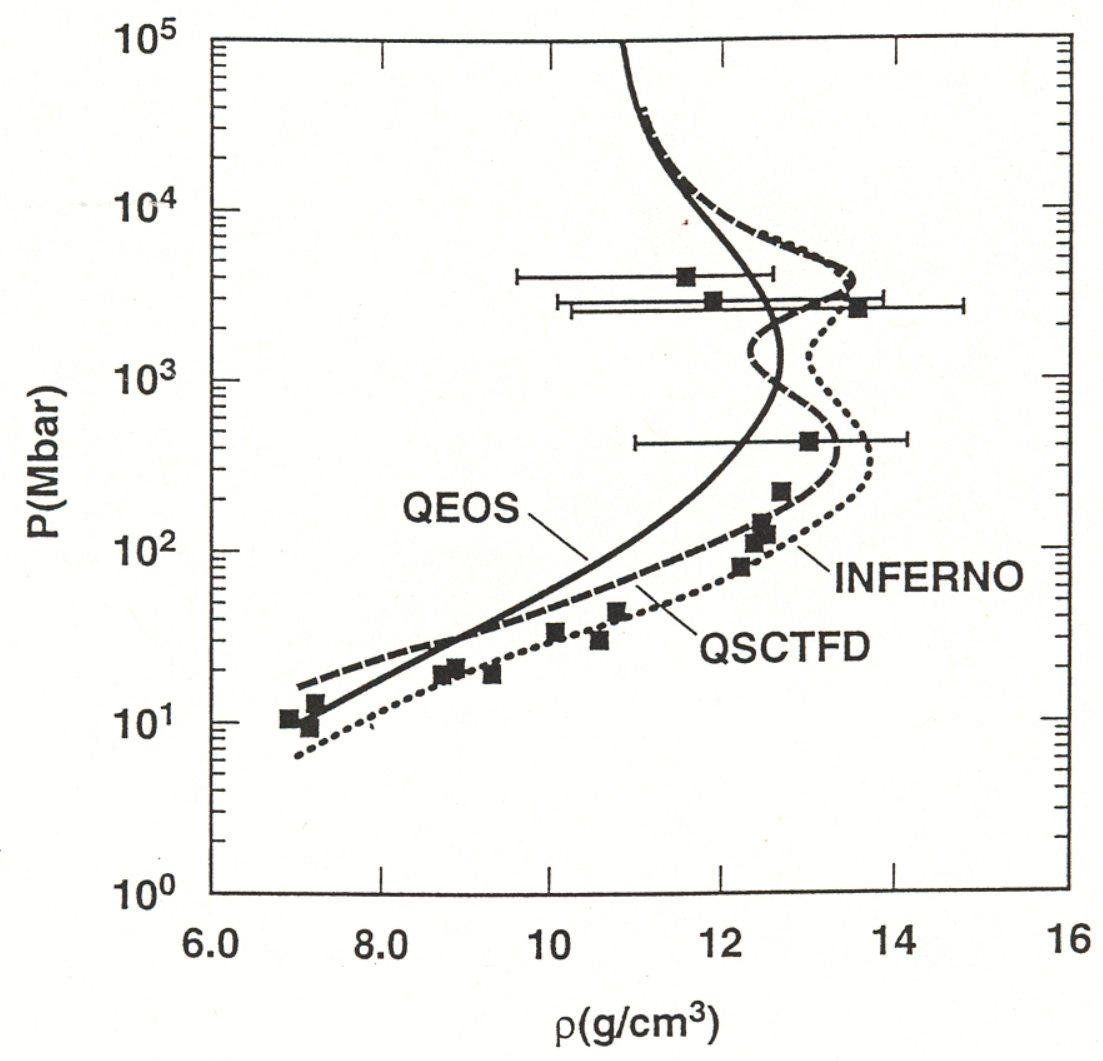

